\title{
MULTIPLIERS OF INVARIANT SUBSPACES IN THE BIDISC
}

\author{
by TAKAHIKO NAKAZI*
}

(Received 23rd January 1992)

\begin{abstract}
For any nonzero invariant subspace $M$ in $H^{2}\left(T^{2}\right)$, set $M^{x}=\left[\bigcup_{n=0}^{\infty} z^{n} M\right] \cap\left[\bigcup_{n=0}^{\infty} \bar{w}^{n} M\right]$. Then $M^{x}$ is also an invariant subspace of $H^{2}\left(T^{2}\right)$ that contains $M$. If $M$ is of finite codimension in $H^{2}\left(T^{2}\right)$ then $M^{x}=H^{2}\left(T^{2}\right)$ and if $M=q H^{2}\left(T^{2}\right)$ for some inner function $q$ then $M^{x}=M$. In this paper invariant subspaces with $M^{x}=M$ are studied. If $M=q_{1} H^{2}\left(T^{2}\right) \cap q_{2} H^{2}\left(T^{2}\right)$ and $q_{1}, q_{2}$ are inner functions then $M^{x}=M$. However in general this invariant subspace may not be of the form: $q H^{2}\left(T^{2}\right)$ for some inner function $q$. Put $\mathscr{H}(M)=\left\{\phi \in L^{\infty}: \phi M \subseteq\right.$ $\left.H^{2}\left(T^{2}\right)\right\}$; then $\mathscr{H}(M)$ is described and $\mathscr{H}(M)=\mathscr{M}\left(M^{x}\right)$ is shown. This is the set of all multipliers of $M$ in the title. A necessary and sufficient condition for $\mathscr{H}(M)=H^{\infty}\left(T^{2}\right)$ is given. It is noted that the kernel of a Hankel operator is an invariant subspace $M$ with $M^{x}=M$. The argument applies to the polydisc case.
\end{abstract}

1980 Mathematics subject classification (1985 Revision): Primary 46J15, 47A15, 47B20; Secondary 32 A35.

\section{Introduction}

Let $T^{2}$ be the torus that is the Cartesian product of 2 unit circles in $\mathbb{C}$. The usual Lebesgue spaces, with respect to the Haar measure $m$ of $T^{2}$, are denoted by $L^{p}=L^{p}\left(T^{2}\right)$, and the Hardy spaces $H^{p}=H^{p}\left(T^{2}\right)$ are spaces of all $f \in L^{p}\left(T^{2}\right)$ whose Fourier coefficients

$$
\hat{f}(j, \ell)=\int_{T^{2}} f(z, w) \bar{z}^{j} \bar{w}^{\ell} d m(z, w)
$$

are 0 as soon as at least one component of $(j, \ell)$ is negative, where $1 \leqq p \leqq \infty$. Let $U^{2}$ be the unit bidisc that is the Cartesian product of 2 open unit discs in $\mathbb{C}$. Any function $f$ in $H^{p}$ has an analytic extension to $U^{2}$ which is also denoted by $f$.

A closed subspace $M$ of $H^{2}$ is said to be invariant if

$$
z M \subset M \text { and } w M \subset M \text {. }
$$

Put

$$
M_{1}=\left[\bigcup_{M=0}^{\infty} \bar{z}^{n} M\right] \text { and } M_{2}=\left[\bigcup_{M=0}^{\infty} \bar{w}^{n} M\right] \text { where }\left[\bigcup_{M=0}^{\infty} \bar{z}^{n} M\right]
$$

*This research was partially supported by Grant-in-Aid for Scientific Research, Ministry of Education. 
is the closed linear span of

$$
\bigcup_{n=0}^{\infty} z^{n} M
$$

in $L^{2}$. Set

$$
M^{x}=M_{1} \cap M_{2} .
$$

Then $M$ is also an invariant subspace of $H^{2}$. Put

$$
\mathbf{H}_{1}=\left[\bigcup_{n=0}^{\infty} z^{n} H^{2}\right] \text { and } \mathbf{H}_{2}=\left[\bigcup_{n=0}^{\infty} \bar{w}^{n} H^{2}\right]
$$

Then $H^{2}=\mathbf{H}_{1} \cap \mathbf{H}_{2}$ and hence $\left(H^{2}\right)^{x}=H^{2}$. Therefore it is desirable to know invariant subspaces $M$ which have the following property: $M^{x}=H^{2}$ or $M^{x}=M$. An invariant subspace $M$ of $H^{2}$ has full range if $M_{1}=\mathbf{H}_{1}$ and $M_{2}=\mathbf{H}_{2}$. Such an invariant subspace has been studied by Agrawal, Clark and Douglas [2]. It is clear that $M$ has full range if and only if $M^{x}=H^{2}$. An invariant subspace $M$ with $M^{x}=M$ has not been studied. In this paper we study invariant subspaces with $M^{x}=M$. For an invariant subspace $M$ of $H^{2}$ set

$$
\mathscr{M}(M)=\left\{\phi \in L^{\infty}: \phi M \subseteq H^{2}\right\} .
$$

It is essentially known [2] that if $M^{x}=M$ then $\mathscr{M}(M)=H^{\infty}$. In this paper we give a necessary and sufficient condition for $\mathscr{M}(M)=H^{\infty}$.

Let $K_{0}^{2}$ denote the orthogonal complement of $\bar{H}^{2}=\left\{f: f \in H^{2}\right\}$ in $L^{2}$. The invariant subspace $q H^{2}$ for an inner function $q$ is called a Beurling subspace.

The author would like to thank Professor K. Takahashi for his helpful discussions.

\section{Intersection of Beurling subspaces}

A Beurling subspace $M$ satisfies $M^{x}=M$ trivially. In this section we show that the intersections of Beurling subspaces have this property.

Proposition 1. If $M$ is a nonzero invariant subspace of $H^{2}$ then there exist two unimodular functions $Q_{1}$ in $H_{1}$ and $Q_{2}$ in $\mathbf{H}_{2}$ such that $M_{1}=Q_{1} \mathbf{H}_{1}$ and $M_{2}=Q_{2} \mathrm{H}_{2}$, and hence

$$
M^{x}=Q_{1} \mathbf{H}_{1} \cap Q_{2} \mathbf{H}_{2} .
$$

Proof. By [7, pp. 164-165], [ $\left.\bigcup_{M=0}^{\infty} \bar{z}^{n} M\right]=\chi_{E} Q_{1} H_{1}+\left(1-\chi_{E}\right) L^{2}$ where $\chi_{E}$ is a characteristic function of some measurable set $E$ in $T^{2}, \chi_{E} \in \mathbf{H}_{1}$ and $\left|Q_{1}\right|=1$ a.e. Since 
$M \subset H^{2}$ and $\mathbf{H}_{1}$ has no reducing invariant subspaces under the multiplication of $w$, this implies that $M_{1}=Q_{1} \mathbf{H}_{1}$ and $Q_{1} \in \mathrm{H}_{1}$ because $M_{1} \subset \mathrm{H}_{1}$. The same argument shows that $M_{2}=Q_{2} H_{2}$ and $Q_{2} \in H_{2}$.

Theorem 2. Let $\ell$ be a finite positive integer. If $M=\bigcap_{j=1}^{\ell} q_{j} H^{2}$ and $\left\{q_{j}\right\}_{j=1}^{\ell}$ are inner functions then $M^{x}=M$. Moreover

$$
M^{x}=Q_{1} \mathbf{H}_{1} \cap Q_{2} \mathbf{H}_{2}
$$

where $\left\{Q_{i}\right\}_{i=1}^{2}$ are unimodular functions in $\left\{\mathbf{H}_{i}\right\}_{i=1}^{2}$ and

$$
M_{i}=Q_{i} \mathbf{H}_{i}=\bigcap_{j=1}^{\ell} q_{j} \mathbf{H}_{i} \quad(i=1,2) .
$$

Proof. Let $N=\bar{M}^{\perp}$, that is, $N$ is the orthogonal complement of $\bar{M}=\{\bar{f}: f \in M\}$. Then $N=\left[\sum_{j=1}^{l} q_{j} K_{0}^{2}\right]$ because $\left(\bar{H}^{2}\right)^{\perp}=K_{0}^{2}$. Put $N_{1}=\left[\sum_{j=1}^{\ell} q_{j} w \mathbf{H}_{1}\right]$ and $N_{2}=\left[\sum_{j=1}^{\ell} q_{j} z \mathbf{H}_{2}\right]$; then $\left\{N_{i}\right\}_{i=1}^{2}$ are invariant subspaces and $N=\left[N_{1}+N_{2}\right]$ because $K_{0}^{2}=w \mathbf{H}_{1}+z \mathbf{H}_{2}$. It is clear that $z N_{1}=N_{1}$ and $w N_{2}=N_{2}$. If $w N_{1}=N_{1}$ then $N_{1} \supset \bar{q}_{j} \bar{w}^{n} \mathbf{H}_{1}$ for any positive $n$ and so $N_{1}=L^{2}$. This contradicts that $M \supset\left(\prod_{j=1}^{t} q_{j}\right) H^{2}$. Thus $w N_{1} \neq N_{1}$ and similarly $z N_{2} \neq N_{2}$. By [7] there exist unimodular functions $Q_{1}$ and $Q_{2}$ such that $N_{1}=Q_{1} w \mathrm{H}_{1}$ and $N_{2}=Q_{2} z \mathbf{H}_{2}$. Since $N=\left[N_{1}+N_{2}\right]$,

$$
M=Q_{1} \mathrm{H}_{1} \cap Q_{2} \mathrm{H}_{2} .
$$

Since $Q_{i} H_{i}$ is the orthogonal complement of $N_{i}$ and $N_{i}=\left(\bar{M}_{i}\right)^{\perp}$ for $i=1,2$,

$$
M_{i}=Q_{i} \mathbf{H}_{i}=\bigcap_{j=1}^{l} q_{j} \mathbf{H}_{i} \quad(i=1,2) .
$$

In the case of one variable an intersection of two Beurling subspaces is also a Beurling subspace. This is not true in the case of two variables by [11, Theorem 2 and its proof]. In fact $X_{1}$ and $X_{2}$ in [11, Theorem 2] are intersections of two Beurling subspaces and $X_{j} \subseteq H^{2}$ for $j=1,2$. If $X_{1}=q_{1} H^{2}$ and $X_{2}=q_{2} H^{2}$ for some inner functions $q_{1}, q_{2}$ then $\bar{q}_{1} X_{1}=\bar{q}_{2} X_{2}=H^{2}$. This contradicts [11, Theorem 2]. Hence our Theorem 2 is not trivial. If an invariant subspace $M$ is determined by vanishing conditions at finitely many points of $U$ then $M^{x}=M$ because $M$ is a finite co-dimensional subspace of $H^{2}$. We are interested in an invariant subspace determined by vanishing conditions at infinitely many points of $U$. Let $s$ be an analytic function on $U$ such that $s(U) \subset U$. Put

$$
M_{s}=\left\{f \in H^{2}: f(z, s(z))=0 \text { for all } z \in U\right\}
$$

then $M_{s}$ is an invariant subspace of $H^{2}$.

Proposition 3. Let $s$ be an analytic function on $U$ and $s(U) \subset U$. Then 


$$
M_{s}^{x}=Q \mathbf{H}_{1} \cap \mathbf{H}_{2}
$$

where $Q=w-s / 1-\vec{s} w$. (1) If $|s|=1$ a.e. on $T$ then $M_{s}^{x}=H^{2}$. (2) If $|s|=r<1$ a.e. on $T$ for some constant $r$ then

$$
M_{s}^{x}=M_{s}=Q^{\prime} \mathbf{H}_{1} \cap \mathbf{H}_{2}=Q^{\prime} H^{2} \cap H^{2}
$$

where $Q^{\prime}=w-s / s r^{-1}-r w$.

Proof. If $f \in M_{s}$ then, for a.e. $z \in T$,

$$
\frac{1-\bar{s} w}{w-s} f(z, w)
$$

is $w$-analytic and hence $f$ belongs to $Q H_{1}$. Therefore $M_{s, 1} \subset Q H_{1}$. Since $(1-r \vec{s} w)^{-1} \in \mathbf{H}_{1}$ for any constant $r$ with $0<r<1$ and $w-s \in M_{s},(w-s)(1-r \bar{s} w)^{-1}$ belongs to $M_{s, 1}$. Since

$$
\left|\frac{w-s}{1-r \bar{s} w}-\frac{w-s}{1-\bar{s} w}\right| \leqq\left|\frac{1-r}{1-r \bar{s} w}\right| \rightarrow 0 \quad(\text { as } r \rightarrow 1)
$$

because $m\left\{(z, w) \in T^{2}: \bar{s}(z) w=1\right\}=0, Q$ belongs to $M_{s, 1}$ and hence $M_{s, 1}=Q H_{1}$. Since $(w-r s)^{-1} \in \mathbf{H}_{2}$ for any $r$ with $0<r<1$ and $w-s \in M_{s},(w-s)(w-r s)^{-1} \in M_{s, 2}$. As $r \rightarrow 1$ the constant 1 belongs to $M_{s, 2}$ and hence $M_{s, 2}=\mathbf{H}_{2}$. This implies $M_{s}^{x}=Q H_{1} \cap \mathbf{H}_{2}$. (1) is clear because $s \mathbf{H}_{1}=\mathbf{H}_{1}$. (2) Since

$$
\frac{w-s}{1-\bar{s} w}=\frac{s r^{-1}(w-s)}{s r^{-1}-r w}, \quad M_{s}=Q^{\prime} \mathbf{H}_{1} \cap \mathbf{H}_{2} .
$$

Put $N=Q^{\prime} H^{2} \cap H^{2}$. If $Q^{\prime} f=g$ for some $f$ and $g$ in $H^{2}$ then

$$
(w-s) f(z, w)=\left(s r^{-1}-r w\right) g(z, w) \quad(z, w) \in U^{2} .
$$

Thus $g$ belongs to $M_{s}$ and hence $M_{s} \supset N$. It is clear that $N_{1} \subset Q^{\prime} \mathbf{H}_{1}$ and $N_{2} \subset \mathbf{H}_{2}$. The $Q^{\prime}$ belongs to $N_{1}$ because $\left(s r^{-1}-r w\right)^{-1} \in H_{1}$ and $w-s \in N$. The constant 1 belongs to $N_{2}$ because $(w-s)^{-1} \in \mathbf{H}_{2}$ and $w-s \in N$. Thus $N=Q^{\prime} \mathbf{H}_{1} \cap \mathbf{H}_{2}$. Since $N^{x}=N$ by the proof of Theorem 2, $M_{s}^{x}=M_{s}=Q^{\prime} \mathbf{H}_{1} \cap \mathbf{H}_{2}=Q^{\prime} H^{2} \cap H^{2}$.

\section{Multipliers of an invariant subspace}

An invariant subspace $M$ of $H^{2}$ is said to be podal if every invariant subspace $N$ of $H^{2}$ which is unitarily equivalent to $M$ is a subspace of $M$ (cf. [3]). Agrawal, Clark and Douglas [2] showed that if an invariant subspace of $H^{2}$ has full range then it is podal. The following is a generalization of that. For if $N$ is full range then $N^{x}=H^{2}$. 
Proposition 4. Let $M$ and $N$ be invariant subspaces of $H^{2}$ with $M \subset N^{x}$. If $M$ is unitarily equivalent to $N$, then $M \subset N$.

Proof. By [2, Lemma 1] $M=q N$ for some unimodular function $q$. Then $M^{x}=q N^{x}$ and $M^{x} \subset N^{x}$. By [2, Proposition 3] the $q$ is an inner function.

Proposition 5. Let $M$ and $N$ be invariant subspaces of $H^{2}$ with $M^{x}=N^{x}$. Then $M$ is unitarily equivalent to $N$ if and only if $M=N$.

Proof. If $M$ is unitarily equivalent to $N$ then $M=q N$ for some unimodular function $q$. Then $M^{x}=q N^{x}$ and hence $M^{x}=q M^{x}$. Thus $q$ is an inner function. By the same argument $N^{x}=\bar{q} N^{x}$ and hence $\bar{q}$ is also an inner function. Thus $q$ is constant and $M=N$.

If $M$ and $N$ are invariant subspaces of finite codimension in $H^{2}$, then we can show $M^{x}=N^{x}$ and hence by Proposition $5 M=N$. This is Corollary 3 in [2] (see [5, Corollary 6]). Douglas and Yan [3] asked the following question. Can one characterize podal invariant subspaces?

Proposition 6. Let $M$ be a nonzero invariant subspace of $H^{2} . M$ is podal if and only if any unimodular functions in $\mathscr{M}(M)$ belong to $H^{\infty}$.

Proof. If $\phi$ is a unimodular function in $\mathscr{M}(M)$ then $\phi M \subset H^{2}$ and $\phi M$ is unitarily equivalent to $M$. If $M$ is podal then $\phi M \subset M$ and hence $\phi \in H^{\infty}$ by [2, Proposition 3]. Suppose any unimodular functions in $\mathscr{M}(M)$ belong to $H^{\infty}$. If $M$ is unitarily equivalent to $N$ which is an invariant subspace in $H^{2}$ then $N=\phi M$ for some unimodular function $\phi$. Since $\phi \in \mathscr{M}(M)$, by the hypothesis $\phi$ belongs to $H^{\infty}$ and so $M$ is podal.

By the proposition above, if $\mathscr{M}(M)=H^{\infty}$ then $M$ is podal. We will characterize an invariant subspace $M$ with $\mathscr{M}(M)=H^{\infty}$. In general $\mathscr{M}(M)$ is an invariant subspace of $L^{\infty}$ which contains $H^{\infty}$. The strusture of $\mathscr{M}(M)$ is simpler than that of $M$.

Theorem 7. If $M$ is a nonzero invariant subspace of $H^{2}$ then

$$
\mathscr{M}(M)=\bar{Q}_{1} \mathbf{H}_{1} \cap \bar{Q}_{2} \mathbf{H}_{2} \cap L^{\infty}
$$

where $M^{x}=Q_{1} \mathbf{H}_{1} \cap Q_{2} \mathbf{H}_{2}$ and $Q_{i}$ is a unimodular function in $\mathbf{H}_{i}$ for $i=1,2$. Hence $\mathscr{H}(M)=H^{\infty}$ if and only if

$$
\bar{Q}_{1} \mathbf{H}_{1} \cap \bar{Q}_{2} \mathbf{H}_{2} \cap L^{\infty}=H^{\infty}
$$

Proof. If $\phi \in \mathscr{M}(M)$ then $\phi M \subset H^{2}$. Hence for $i=1,2$

$$
\phi M_{i} \subset \mathbf{H}_{i} \text { and } M_{i}=Q_{i} \mathbf{H}_{i}
$$


where $Q_{i}$ is a unimodular function in $\mathbf{H}_{i}$. Thus $\phi \in \bar{Q}_{1} \mathbf{H}_{1} \cap \bar{Q}_{2} \mathbf{H}_{2} \cap L^{\infty}$ and hence $\mathscr{M}(M) \subset \bar{Q}_{1} \mathbf{H}_{1} \cap \bar{Q}_{2} \mathbf{H}_{2} \cap L^{\infty}$. Conversely let $\phi$ be in $\bar{Q}_{1} \mathbf{H}_{1} \cap \bar{Q}_{2} \mathbf{H}_{2} \cap L^{\infty}$. Then

$$
\phi=\bar{Q}_{1} \phi_{1}=\bar{Q}_{2} \phi_{2}
$$

where $\phi_{i}$ is in $\mathbf{H}_{i}$ for $i=1,2$. If $f \in M$ then $f \in M^{x}$ and hence

$$
f=Q_{1} f_{1}=Q_{2} f_{2}
$$

where $f_{i}$ is in $\mathbf{H}_{i}$ for $i=1,2$. Therefore $\phi f=\phi_{1} f_{1}=\phi_{2} f_{2}$. This implies that $\phi f \in \mathbf{H}_{1} \cap$ $\mathbf{H}_{2}=H^{2}$ and hence $\phi \in \mathscr{M}(M)$. Thus

$$
\bar{Q}_{1} \mathbf{H}_{1} \cap \bar{Q}_{2} \mathbf{H}_{2} \cap L^{\infty} \subset \mathscr{M}(M) .
$$

This establishes the theorem.

For $\phi \in L^{\infty}$, the Hankel operator determined by $\phi$ is

$$
H_{\phi}=(1-P) M_{\phi} \mid H^{2}
$$

where $P$ is the orthogonal projection from $L^{2}$ to $H^{2}$.

Proposition 8. The kernel of a Hankel operator is an invariant subspace $M$ with $M^{x}=M$.

Proof. Suppose $H_{\phi}$ is the Hankel operator defined by $\phi \in L^{\infty}$ and let $M$ be its kernel. It is clear that $M$ is an invariant subspace. Since $M$ is the kernel of $H_{\phi}, \phi M \subset H^{2}$. By Theorem 7, $\mathscr{M}(M)=\mathscr{M}\left(M^{x}\right)$ and $\phi M^{x} \subset H^{2}$. This implies $M=M^{x}$ because $M=$ $\left\{f \in H^{2}: \phi f \in H^{2}\right\}$.

\section{REFERENCES}

1. P. R. Ahern and D. N. Clark, Invariant subspaces and analytic continuation in several variables. J. Math. Mech. 19 (1970), 963-969.

2. O. P. Agrawal, D. N. Clark and R. G. Douglas, Invariant subspaces in the polydisk, Pacific J. Math. 121 (1986), 1-11.

3. R. G. Douglas and K. YAN, On the rigidity of Hardy submodules, Integral Equations Operator Theory 13 (1990), 350-363.

4. K. Hoffman, Banach Spaces of Analytic Functions. (Prentice-Hall, 1962).

5. K. Izuchi, Unitary equivalence of invariant subspaces in the polydisk, Pacific J. Math. 130 (1987), 351-358.

6. T. NAKAZI, Nonmaximal weak-* Dirichlet algebra, Hokkaido Math. J. 5 (1976), 88-96.

7. T. NakAzı, Invariant subspaces of weak-* Dirichlet algebras. Pacific J. Math. 69 (1977), 151-167. 
8. T. Nakazı, Certain invariant subspaces of $H^{2}$ and $L^{2}$ on a bidisc, Canad. J. Math. 40 (1988), 1272-1280.

9. T. Nakazı, Invariant subspaces in the bidisc and commutators, J. Austral. Math. Soc., to appear.

10. T. NaKazl, Homogeneous polynomials and invariant subspaces in the polydiscs. Arch. Math. 58 (1992). 56-63.

11. W. Rudin, Invariant subspaces of $H^{2}$ on a torus, J. Funct. Anal. 61 (1985), 378-384.

Department of Mathematics

FACULTY OF SCIENCE

HoKkaIdo UnIVERSITY

SAPPORO 060

JAPAN 